\section{Rehabilitación pulmonar en cáncer pulmonar}

\section{Pulmonary rehabilitation in lung cancer}

Elena M. Chevalier Chockee, MD¹ Olga Milena García, MD²

\section{Resumen}

El cáncer pulmonar es una enfermedad que conlleva una enorme carga económica y social. La rehabilitación pulmonar ha surgido como una intervención costo-efectiva y ha demostrado mejorar la capacidad de ejercitarse, los síntomas y la calidad de vida en aquellos con enfermedades pulmonares crónicas, principalmente la enfermedad pulmonar obstructiva crónica (EPOC). Hay un mayor reconocimiento de que la rehabilitación pulmonar puede beneficiar a otras condiciones respiratorias como el cáncer pulmonar primario y metastásico a pulmón, en el cual se han documentado efectos positivos en los pacientes; sin embargo, su rol en el ámbito del tratamiento integral no ha sido bien descrito debido a investigaciones limitadas hasta la fecha.

La rehabilitación pulmonar ha demostrado en pacientes con cáncer pulmonar mejorar la fuerza, la resistencia y la capacidad de ejercitarse en cualquier estadio de la enfermedad. El componente de entrenamiento de la rehabilitación en el preoperatorio y postoperatorio mejora el consumo de oxígeno preoperatorio, la capacidad de ejercitarse y la calidad de vida; y reduce los síntomas, las complicaciones postoperatorias y la duración de la estancia hospitalaria.

En los pacientes que son candidatos a manejo quirúrgico aún no se ha establecido el tiempo óptimo para iniciar la rehabilitación pulmonar ni la duración del programa, pero algunos estudios han mostrado que un programa de 4 a 6 semanas prepara adecuadamente para la cirugía a aquellos pacientes con cáncer pulmonar y EPOC, reduciendo las limitaciones funcionales.

Los cuidados paliativos son un abordaje comprensivo a largo plazo que puede ser paralelo o integrado al tratamiento del cáncer, mientras que la rehabilitación pulmonar es una estrategia que impacta desenlaces específicos.

Palabras clave: rehabilitación pulmonar, cáncer pulmonar, ejercicios de entrenamiento.

\begin{abstract}
Lung cancer is a devastating disease that carries tremendous social and economic burden. Pulmonary rehabilitation has emerged as a cost-effective intervention and has been proven to improve exercise capacity, symptoms and quality of life of patients with chronic lung diseases, particularly chronic obstructive pulmonary disease (COPD). There is also increased recognition that pulmonary rehabilitation can benefit patients with other respiratory conditions such as primary lung cancer and metastatic lung cancer, in which positive effects have been documented in patients. However, their role in the field of comprehensive treatment has not been well described due to limited investigations to date.
\end{abstract}

${ }^{1}$ Fellow de Neumología, Universidad de Panamá, Hospital Santo Tomás. Ciudad de Panamá, Panamá.

${ }^{2}$ Neumólogo, Unidad de Neumología, Departamento de Medicina Interna, Hospital Universitario San Ignacio. Bogotá D. C., Colombia.

Autor de correspondencia

Elena M. Chevalier Chockee. Correo electrónico: emcc83@yahoo.com. Recibido: 25/6/2018. Aceptado: 15/11/2018. 
Pulmonary rehabilitation has shown in patients with lung cancer to improve strength, resistance and exercise capacity at any stage of disease. The training component of the rehabilitation program in the preoperative and postoperative improves preoperative oxygen consumption, exercise capacity and quality of life; and also reduces the symptoms, postoperative complications and length of hospital stay.

The optimum time to initiate pulmonary rehabilitation and the duration of the program in patients who are candidates for surgical management are not yet established, but studies have shown that a 4- to 6-week program adequately prepares those patients with lung cancer and COPD for surgery, reducing functional limitations.

Palliative care is a comprehensive long-term approach that can be parallel or integrated to cancer treatment, while pulmonary rehabilitation is a strategy that impacts specific outcomes.

Keywords: Pulmonary rehabilitation, lung cancer, training exercises.

\section{Introducción}

El cáncer pulmonar es una enfermedad que conlleva una enorme carga económica y social, es en la actualidad uno de los canceres más prevalentes y permanece como la principal causa de muerte relacionada con cáncer a nivel mundial a pesar de los avances en las modalidades de tratamiento (1). La supervivencia a los 5 años es pobre, pero la detección temprana, la terapia dirigida y el abordaje multidisciplinario para el diagnóstico y manejo contribuyen a mejorar el pronóstico. Uno de los componentes claves en estos pacientes es considerar las comorbilidades relacionadas con el tabaquismo, las complicaciones relacionadas con el tratamiento (quimioterapia, radioterapia) y los riesgos de complicaciones posquirúrgicas (2).

La rehabilitación pulmonar ha surgido como una intervención costo-efectiva y ha demostrado mejorar la capacidad de ejercitarse, los síntomas y la calidad de vida en aquellos con enfermedades pulmonares crónicas, principalmente la enfermedad pulmonar obstructiva crónica (EPOC). Tanto la EPOC como las enfermedades pulmonares intersticiales coexisten con el cáncer pulmonar, cuya incidencia es del 8,5\% y del $6 \%-15$ $\%$, respectivamente (2).

Hay un mayor reconocimiento de que la rehabilitación pulmonar puede beneficiar a otras condiciones respiratorias como las enfermedades pulmonares intersticiales, hipertensión pulmonar, cáncer pulmonar primario y metastásico a pulmón, en el cual se han do- cumentado efectos positivos en los pacientes; sin embargo, su rol en el ámbito del tratamiento integral no ha sido bien descrito debido a investigaciones limitadas hasta la fecha.

\section{Rehabilitación pulmonar}

La rehabilitación pulmonar es una intervención multidisciplinaria basada en la evidencia, dirigida a pacientes con enfermedades respiratorias crónicas sintomáticas. Se considera un elemento clave para el cuidado integral, definido como una intervención basada en una evaluación sistemática del paciente que culmina en una terapia individualizada. Las opciones terapéuticas incluyen ejercicios de entrenamiento, educación y cambios conductuales, con los que se busca mejorar la condición física, psicológica y la calidad de vida de aquellos con enfermedades respiratorias crónicas, y generar cambios que promuevan la adherencia a largo plazo y las conductas saludables. En el caso particular del cáncer, la rehabilitación pulmonar también proporciona la oportunidad de adaptarse a nuevas estrategias terapéuticas como quimioterapia, trasplante pulmonar, cirugía de reducción del volumen pulmonar y ventilación mecánica no invasiva $(2,3)$.

Un protocolo estándar consta de 3 sesiones de 30 a 90 minutos por semana por 6-8 semanas, que consiste en ejercicios aeróbicos individualizados y entrenamientos de fuerza. Un programa de 12 semanas logra mayores beneficios y se mantienen los efectos hasta los 12-18 meses. La rehabilitación pulmonar también inte- 
gra un componente nutricional, cesación del consumo de tabaco, ejercicios respiratorios, intervenciones psicosociales y educación (2).

La eficacia está bien establecida en el manejo de pacientes con EPOC, logrando beneficios como el incremento en la distancia de la caminata de 6 minutos, mejoría de la fuerza muscular de los miembros superiores e inferiores, mejoría de la calidad de vida, reducción de las exacerbaciones y disminución en la estancia hospitalaria. Independientemente de si los ejercicios son supervisados o no, son capaces de mantener la calidad de vida y la tolerancia al ejercicio a los 12 meses. La repetición de un ciclo de rehabilitación pulmonar no se requiere anualmente a menos que presente un aumento de los síntomas o exacerbaciones. Estos ciclos mejoran los beneficios a corto plazo sin efecto aditivo, independientemente del intervalo de duración entre cada ciclo de rehabilitación pulmonar (4-6).

En otras enfermedades como la enfermedad restrictiva pulmonar e hipertensión pulmonar que reciben tratamiento médico se ha demostrado mejoría en la capacidad de ejercitarse y la calidad de vida, por lo que otras condiciones como el cáncer pulmonar se pueden beneficiar de la mejoría en la fuerza muscular y en la calidad de vida $(2,3)$.

\section{Carga del cáncer pulmonar en la calidad de vida y la capacidad de ejercitarse}

El cáncer pulmonar es uno de los canceres más prevalentes y permanece como la principal causa de muerte relacionada con cáncer a nivel mundial a pesar de los avances en las modalidades de tratamiento. El cáncer de células no pequeñas es el más común, que corresponde al $85 \%$ de casos, y el $15 \%$ corresponde al de células pequeñas. Aproximadamente el $40 \%$ de los pacientes con cáncer de células no pequeñas llevados a resección pulmonar completa del tumor primario sobrevive a los 5 años. En cambio, en el cáncer de células pequeñas la metástasis es común al momento del diagnóstico y la resección pulmonar es rara vez una opción $(3,7)$.

La manifestación clínica del cáncer de pulmón primario depende de la localización. Generalmente se presenta tos, disnea, dolor pleurítico y pérdida de peso. La disnea tiene una alta prevalencia: del $55 \%-87 \%$ en todos los estadios de la enfermedad, asociado con otros síntomas como fatiga, ansiedad, depresión e insomnio. La inactividad física es común, sobre todo en etapas avanzadas, debido a los síntomas relacionados con la enfermedad y los efectos adversos del tratamiento. A pesar del incremento en la terapia dirigida en cáncer pulmonar, pocos abordajes terapéuticos alivian la carga de síntomas, los cuales alteran de manera significativa la calidad de vida (3).

La persistencia de la fatiga posterior al tratamiento es común en estos pacientes, llevándolos a un ciclo vicioso de inactividad y desacondicionamiento, lo que impide la recuperación del tratamiento (8).

La proteólisis y lipólisis inducida por cáncer condiciona la pérdida de peso y el desgaste muscular, lo que conlleva a que presenten desacondicionamiento con debilidad de los músculos esqueléticos, intolerancia al ejercicio y discapacidad funcional.

El tratamiento en estos pacientes conlleva también a una disminución en la capacidad de ejercicio, 6 meses después de la resección pulmonar (lobectomía o neumonectomía) se ha documentado una disminución del consumo de oxígeno pico de un $13 \%$ a $28 \%$ y de la función pulmonar (volumen espiratorio forzado el primer segundo $\left[\mathrm{VEF}_{1}\right]$, capacidad vital forzada $[\mathrm{CVF}]$, capacidad pulmonar total [CPT], capacidad de difusión del monóxido de carbono [DLCO]). La terapia adyuvante con quimioterapia favorece el desacondicionamiento, que posteriormente reduce la capacidad de entrega o utilización de oxígeno y el sustrato durante el ejercicio, lo que contribuye a la intolerancia al ejercicio (7).

Estos pacientes tienen alta incidencia de enfermedades respiratorias crónicas concomitantes, las cuales contribuyen a una mayor alteración ventilatoria o al intercambio gaseoso, agravando aún más los síntomas.

Adicionalmente, estudios recientes han mostrado que de los pacientes con cáncer pulmonar de reciente diagnóstico, hasta un $73 \%$ de los hombres y $53 \%$ de las mujeres tienen como comorbilidad la EPOC (3).

El estado funcional determina las decisiones terapéuticas y puede excluir a los pacientes de recibir una terapia específica. Una baja tolerancia al ejercicio está asociada con un peor pronóstico posquirúrgico, res- 
puesta subóptima a la quimioterapia y reducción de la supervivencia. La fisioterapia del tórax en conjunto con los ejercicios de entrenamiento ha demostrado reducir los síntomas, incrementar la tolerancia al ejercicio y mejorar la calidad de vida (2).

Debido a las mejoras en las técnicas quirúrgicas y el incremento en la precisión y calidad de las terapias adyuvantes, el pronóstico de los pacientes con cáncer pulmonar está mejorando. La calidad de vida, fatiga y capacidad de ejercicio son de importancia clínica significativa en el tratamiento de cáncer pulmonar de células no pequeñas.

\section{Rehabilitación pulmonar en cáncer pulmonar}

En la revisión por H. Wong y colaboradores se menciona la definición dada por Silver: la rehabilitación en cáncer se define como los cuidados médicos que se deben integrar a través de cuidados oncológicos continuos guiados por profesionales de rehabilitación entrenados y que tienen dentro de su ámbito de práctica para diagnosticar y tratar alteraciones físicas, psicológicas y cognitivas en un esfuerzo para mantener o restaurar las funciones, reducir la carga de los síntomas, maximizar la independencia y mejorar la calidad de vida (3).
La rehabilitación pulmonar logra mejorar significativamente el consumo de oxígeno máximo, lo cual permite que el paciente tenga criterios de elegibilidad para cirugía y tener una resección exitosa. Aunque el tipo de cáncer es un contribuidor importante de los resultados de salud y bienestar en estos pacientes que reciben rehabilitación pulmonar, dichos estudios aún no se han realizado. Pocos ensayos sugieren que los ejercicios de entrenamiento son seguros y factibles en aquellos con cáncer pulmonar en estadios avanzados y que el incremento en la actividad física puede mejorar la tolerancia al ejercicio y la carga de síntomas; sin embargo, los regímenes han sido diversos en cuanto a la localización, duración e intensidad del ejercicio; por tanto, el régimen óptimo para recomendar es incierto (3).

\section{Principios de rehabilitación pulmonar en cáncer}

La rehabilitación pulmonar para pacientes con cáncer pulmonar tiene 3 elementos claves: un plan individualizado para cada paciente, un equipo multidisciplinario y el reconocimiento de todos los elementos de la enfermedad (Figura 1).

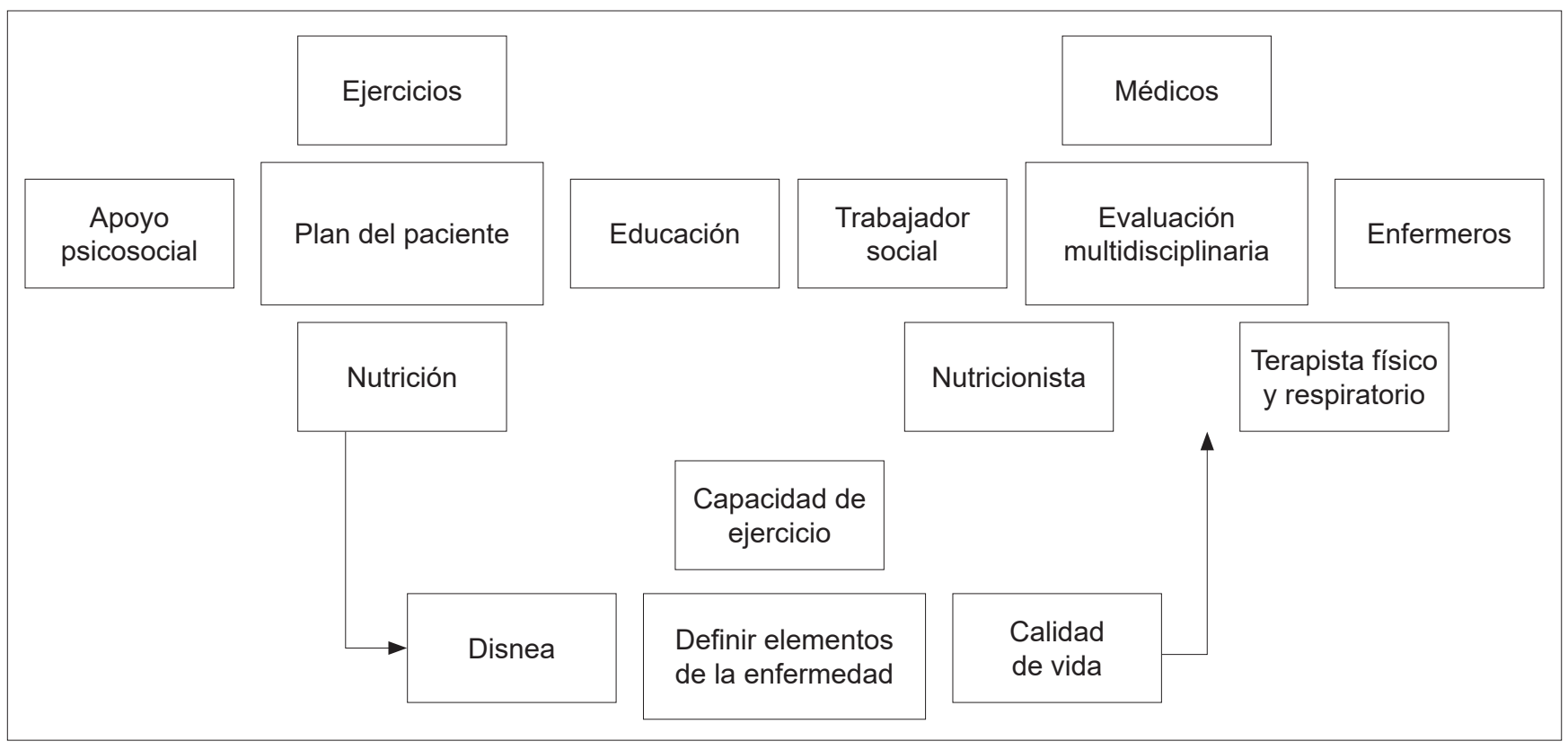

Figura 1. Componentes claves de la rehabilitación pulmonar en pacientes con cáncer pulmonar. Adaptado de: Wang $\mathrm{H}$ et al. 2016;8(10):990-6. 
Una evaluación integral de estos pacientes es decisiva para desarrollar un plan de cuidados individualizado y apropiado. La capacidad de ejercitarse, calidad de vida y disnea se deben evaluar utilizando herramientas de medición específicas para guiar la prescripción del ejercicio y evaluar la efectividad del programa. Es muy importante evaluar en estos pacientes el aspecto psicológico, emocional y social (3).

\section{Componentes de la rehabilitación pulmonar}

\section{Ejercicios de entrenamiento}

Los ejercicios de entrenamiento mejoran la fuerza, resistencia y capacidad de ejercitarse, y son aplicables en cualquier estadio de la enfermedad; sin embargo, se deben prescribir con consideración en la carga, progresión, especificidad y reversibilidad.

En cuanto a la duración del entrenamiento, este varía de 4 a 12 semanas en los diferentes estudios y se ha observado que las intervenciones cortas han reportado resultados positivos durante la hospitalización, en casa y en el postoperatorio. En pacientes con cáncer de células no pequeñas, 8 semanas de ejercicios aeróbicos mejoran la capacidad de ejercicio, alivian la fatiga y la disnea. Según H. Wang y colaboradores, un entrenamiento de 12 semanas consistente en caminata diaria en casa logra mejorar la depresión y ansiedad en sobrevivientes de cáncer pulmonar. En pacientes con cáncer de células pequeñas y enfermedad extensa, 6 semanas de un programa de ejercicio estructurado hospitalario lleva a una mejoría en la capacidad funcional, ansiedad y bienestar emocional (9). Inclusive aquellos que reciben tratamiento (quimioterapia y radioterapia) en cáncer de células no pequeñas, luego de la rehabilitación pulmonar se observó una mejoría en la función pulmonar $\left(\mathrm{CVF}, \mathrm{VEF}_{1}\right)$ e incremento en la presión arterial de oxígeno y la saturación de oxígeno (3).

Los ejercicios de entrenamiento pre- y postoperatorios mejoran el consumo de oxígeno preoperatorio, la capacidad de ejercitarse, la calidad de vida y la reducción de los síntomas, así como la morbilidad postoperatoria (3).

\section{Educación}

Es un elemento vital de todo programa de rehabilitación pulmonar. Una educación estructurada mejora el seguimiento de aquellos con regímenes terapéuticos. La misma debe incluir educación en técnicas de aclaramiento de las vías aéreas, respiración diafragmática, respiración segmentaria, relajación muscular progresiva y manejo de los síntomas.

La fatiga, ansiedad y disnea se consideran un conjunto de síntomas que ocurren frecuentemente de manera simultánea en pacientes con cáncer pulmonar avanzado. Dentro de la revisión de H. Wang, los autores Wangnum y colaboradores, en un ensayo controlado aleatorizado, demostraron que un programa educativo multidisciplinario en autocuidado disminuye la fatiga en pacientes con cáncer que reciben quimioterapia (3).

La educación puede mejorar la recuperación posquirúrgica para múltiples procedimientos, proporcionando beneficios en los resultados postoperatorios. La educación en fisioterapia preoperatoria puede disminuir las complicaciones y acortar la estancia hospitalaria asociada con el procedimiento quirúrgico (3).

\section{Soporte nutricional}

La malnutrición está asociada con un pobre pronóstico y mayor riesgo de infecciones en aquellos con cáncer pulmonar luego de la cirugía debido a la supresión de la inmunidad mediada por células y alteración en la cicatrización de las heridas. En estos pacientes hay una reducción en la producción de surfactante y síntesis de proteínas, lo que conlleva a la reducción de la función de los músculos periféricos, la fuerza de los músculos respiratorios, la tolerancia al ejercicio y la calidad de vida. El bienestar nutricional es importante inclusive en aquellos con remisión o en quienes el cáncer se ha controlado (3).

La pérdida de peso y el desgaste muscular son comunes en pacientes con cáncer pulmonar, e inicia de manera temprana durante el tratamiento quimioterapéutico. Se recomienda que estos pacientes reciban una intervención nutricional intensiva temprana. Un estu- 
dio realizado en un centro australiano demostró que un programa de rehabilitación nutricional de 2 meses que incluye una intervención nutricional individualizada en combinación con ejercicios de entrenamiento mejora el peso, resistencia y fuerza en los pacientes con cáncer. El incremento de la ingesta calórica durante la rehabilitación pulmonar ha resultado en un mejor desempeño en el ejercicio en los pacientes con EPOC (3).

Se han evaluado múltiples suplementos nutricionales como el aceite de pescado (2,2 $\mathrm{g}$ diario), el cual mantiene el peso y la masa muscular en aquellos con cáncer de células no pequeñas durante la quimioterapia (10). Los ácidos grasos poliinsaturados n-3 han demostrado efectos positivos en la calidad de vida, desempeño funcional y actividad física en los pacientes con cáncer de células no pequeñas que reciben tratamiento multimodal. En otro estudio se reportó que el ácido eicosapentaenoico mejora de manera significativa la composición corporal, energía e ingesta de proteínas mientras reduce la fatiga, pérdida de apetito y neuropatía, en aquellos con cáncer de células no pequeñas que reciben quimioterapia (3).

\section{Soporte psicosocial}

$\mathrm{Al}$ igual que los pacientes con EPOC, aquellos con cáncer pulmonar experimentan niveles elevados de ansiedad y depresión. Estos efectos psicológicos pueden aumentar la percepción de la gravedad de la disnea y están asociados con un pobre desempeño social, físico y de la calidad de vida.

Las intervenciones psicosociales y conductuales en rehabilitación pulmonar pueden incluir educación, terapia cognitiva conductual y grupos de apoyo enfocados en el manejo del estrés y relajación muscular progresiva. Los beneficios de estas intervenciones incluyen una mejor apreciación de los cambios físicos y psicológicos asociados con el cáncer pulmonar, mejor autocuidado y adherencia al plan de tratamiento, así como una participación en el tratamiento. Otras estrategias adicionales incluyen la respiración diafragmática y de labios fruncidos, simplificación del trabajo y conservación de energía (3).

\section{Rehabilitación pulmonar previa a cirugía}

La rehabilitación pulmonar previa a la cirugía en aquellos que serán llevados a resección pulmonar (neumonectomía o lobectomía) ha demostrado que incrementa el consumo de oxígeno preoperatorio y la distancia de la caminata de 6 minutos, y disminuye las complicaciones postoperatorias y la duración de la estancia hospitalaria. Combinar la rehabilitación pulmonar con fisioterapia respiratoria lleva a disminuir la incidencia de atelectasias e infecciones nosocomiales. En pacientes con EPOC y cáncer de células no pequeñas se evaluó el impacto de la rehabilitación pulmonar en complicaciones postoperatorias y se encontró en un análisis retrospectivo que la misma contribuye a reducir las complicaciones pulmonares postoperatorias (neumonía, fuga del aérea prolongada y neumotórax) luego de la lobectomía (11).

Independientemente del tipo de abordaje quirúrgico, ya sea por toracotomía o videotoracoscopia, un programa de rehabilitación pulmonar preoperatorio es adecuado. En un estudio realizado en España se evaluó el impacto de este sobre el rendimiento funcional de los pacientes sometidos a cirugía torácica asistida por video debido a neoplasia maligna pulmonar y se encontró una mejoría significativa en la capacidad funcional y fuerza muscular (12).

En un estudio retrospectivo en el que se evaluó el impacto de la rehabilitación pulmonar preoperatoria en aquellos con cáncer pulmonar y función pulmonar disminuida $\left(\mathrm{VEF}_{1}<1,6 \mathrm{~L}\right)$, quienes fueron sometidos a cirugía de resección pulmonar (toracoscopia o toracotomía), se encontró que la rehabilitación pulmonar mejoraba el $\mathrm{VEF}_{1}$ en aquellos con $\mathrm{VEF}_{1}$ disminuido (previo: $1,39 \pm 0,13$; posterior: $1,55 \pm 0,06 ; p=0,02$ ), comparado con el grupo control; sin embargo, en este estudio no se demostró un impacto sobre la estancia hospitalaria, morbilidad o mortalidad. Se considera un medio para optimizar el estado de los pacientes antes de la cirugía y de esta manera podría aumentar el número de candidatos para resección quirúrgica, en el contexto de la mejoría en el consumo de oxígeno $(12,13)$. 
Es un reto implementar la rehabilitación pulmonar en el período entre el diagnóstico de cáncer de células no pequeñas y el tratamiento quirúrgico, ya que se requiere la remoción quirúrgica del cáncer lo antes posible; los estudios han mostrado efectividad en programas de 4 a 6 semanas con mejoría en las limitaciones funcionales. Estudios posteriores deberán establecer el tiempo mínimo de duración del programa sin retrasar el manejo quirúrgico (2).

\section{Rehabilitación pulmonar después de la cirugía}

Después de la cirugía de resección pulmonar, los pacientes son propensos al decline de la capacidad de ejercitarse al mes de la lobectomía con la capacidad de recuperar los niveles basales 1 año después de la cirugía (14). La rehabilitación pulmonar se ha utilizado para prevenir alteraciones significativas en la capacidad de ejercitarse y de síntomas respiratorios luego de la cirugía. En la revisión de H. Rivas Pérez, los autores Spruit y colaboradores demostraron que los pacientes tienen mejoría significativa en la capacidad de ejercitarse luego de 8 semanas de un programa de rehabilitación pulmonar intrahospitalario posterior a la cirugía, sin cambios en las pruebas de función pulmonar (2). Otros han encontrado que un programa de 4 semanas posterior a resección pulmonar reduce la disnea, mejora la distancia en la caminata de 6 minutos y aumenta el $V_{E F}$ y CVF. En la revisión de $\mathrm{H}$. Wang se meniona el estudio de Hoffman y colaboradores, en el que encontraron en 7 pacientes con cáncer de células no pequeñas llevados a toracotomía que un programa iniciado al alta hospitalaria lleva a una mejoría de la capacidad funcional, calidad de vida y reducción de la fatiga asociada con el cáncer (3).

La evidencia de un metaanálisis publicado en 2014 sugiere que los ejercicios de entrenamiento que incluyen ejercicios de resistencia y aeróbicos pueden incrementar la capacidad de ejercitarse después de una cirugía de resección pulmonar en cáncer de células no pequeñas. Aunque la calidad de la evidencia es baja, la referencia se debe considerar en esta población (7).

Un estudio observacional de 12 semanas de rehabilitación pulmonar tuvo efectos positivos en la fatiga de aquellos pacientes con cáncer pulmonar de células no pequeñas en estadio I a IIIa tratados de manera curativa con cirugía. Se encontró una mejoría en la calidad de vida y capacidad de ejercicio pico, así como también una reducción significativa de la fatiga (8).

En cuanto al efecto de un programa de rehabilitación pulmonar postoperatorio en el nivel de actividad física, se encontró en un estudio prospectivo observacional de pacientes con cáncer de células no pequeñas en estadio I/II con menos de 85 años llevados a lobectomía por videotoracoscopia que el decline de la actividad física fue significativamente menor en el grupo de rehabilitación pulmonar comparado con el grupo control $(0,014 \pm 0,007$ frente a $0,123 \pm 0,018 ; p$ $<0,001)$. La rehabilitación pulmonar mejoró el nivel de actividad física a niveles preoperatorios, mientras que el grupo control no (15).

Una desventaja de implementar la rehabilitación pulmonar inmediatamente después de la cirugía es el dolor postoperatorio, por lo que algunos autores sugieren que se debe iniciar al tercer mes del postoperatorio.

La duración óptima de un programa de rehabilitación pulmonar es incierta; sin embargo, aquellas que duran más de 2 semanas han demostrado llevar a mejores resultados (2).

\section{Rehabilitación pulmonar en candidatos no quirúrgicos}

Hay información limitada en cuanto a los beneficios potenciales de la rehabilitación pulmonar en aquellos con cáncer pulmonar avanzado y que no son candidatos a cirugía. La quimioterapia y radioterapia llevan a complicaciones pulmonares significativas como neumonitis, infecciones y mayor riesgo de hospitalizaciones. Las alteraciones en la capacidad pulmonar de base probablemente predigan un incremento en el riesgo de complicaciones producidas por la quimioterapia. De manera concomitante, estos presentan fatiga, caquexia y debilidad, que impactan aún más en la calidad de vida (14).

La rehabilitación pulmonar es una herramienta útil posterior al mes de la terapia de cáncer pulmonar (quirúrgica, quimioterapia, radioterapia o una combinación de estas intervenciones), ya que ha demostrado una 
mejoría en la capacidad de ejercitarse y la mejoría de las pruebas de función pulmonar independientemente de la presencia de EPOC (2).

La eficacia de un programa de rehabilitación pulmonar en pacientes sometidos a quimioterapia se ha evaluado en estudios piloto. La tolerancia al ejercicio, medido por la caminata de 6 minutos, disminuye significativamente después de la quimioterapia para cáncer pulmonar. En pacientes que no son candidatos quirúrgicos se ha evaluado la rehabilitación pulmonar intrahospitalaria y el programa consistió en 4 semanas de ejercicios en banda y bicicleta, en los cuales los pacientes lograron el $80 \%$ del predicho de la capacidad de ejercicio máxima. Se reportó una mejoría estadísticamente significativa en la distancia de la caminata de 6 minutos en aquellos que recibieron rehabilitación pulmonar posterior a quimio- y radioterapia. En un estudio retrospectivo de 47 pacientes con cáncer de células no pequeñas se encontró un incremento de la distancia en la caminata de 6 minutos de 41 metros ( $p$ $<0,001)$ luego del tratamiento (2). De igual manera, se han demostrado beneficios en el desempeño de ejercicios cuando la rehabilitación pulmonar se realizó de manera concomitante con la quimio- y radioterapia. En la revisión de H. Rivas Pérez se referenció un estudio prospectivo de Shannon y colaboradores de 2011, en el que 189 pacientes con cáncer de células no pequeñas tuvieron mayores ganancias en la distancia de la caminata de los 6 minutos $(+92,5$ metros frente a $+64,3$ metros; $p<0,001)(2)$.

\section{Rehabilitación pulmonar y cuidados paliativos}

La rehabilitación pulmonar, como una intervención en la calidad de vida, tiene un rol en los cuidados paliativos de los pacientes con cáncer pulmonar. Ambas son complementarias para optimizar la capacidad funcional y minimizar la carga de síntomas en aquellos con cáncer pulmonar, compartiendo la misma meta: mejorar la calidad de vida. Las intervenciones en la calidad de vida son tan importantes como las terapias dirigidas de la enfermedad. Combinando el arte y las habilidades de los cuidados clínicos y fisiológicos y las herramientas conductuales, la rehabilitación pulmonar puede servir para mejorar la capacidad funcional de aquellos pacientes limitados por la disnea y el desacondicionamiento.

Existen herramientas de la rehabilitación pulmonar que incluyen entrenamiento de la respiración, como la respiración con labios fruncidos para mejorar el intercambio gaseoso y disminuir la disnea; habilidades en el automanejo de manera que mejore la confianza y permita un mejor entendimiento de la enfermedad; técnicas de aclaramiento de vías aéreas, optimización de la broncodilatación, cesación de tabaquismo y terapia de oxígeno (16).

El tratamiento activo del dolor, disnea, insomnio y fatiga son componentes importantes del tratamiento del cáncer pulmonar, y el manejo de estos síntomas es uno de los componentes de los cuidados paliativos. La mayoría de los pacientes presenta disnea al esfuerzo, por lo que la respuesta natural más adaptativa es tornarse inactivo, sedentario y, consecuentemente, desacondicionado. La inactividad es deletérea para estos pacientes, condicionando mayor disnea al intentar hacer un esfuerzo y convirtiéndolo en un ciclo vicioso. El alivio de la disnea es una prioridad compartida tanto de la rehabilitación pulmonar como de los cuidados paliativos. El tratamiento de la disnea tiene varios componentes, ya sea fisiológico, psicológico o una combinación de ambas. A corto plazo se encuentra la meditación, los medicamentos y ejercicios respiratorios; mientras que, a largo plazo, los ejercicios de entrenamiento son altamente efectivos (16).

La fatiga es otro de los síntomas frecuentemente expresados por estos pacientes, es un síntoma angustiante y multidimensional, con un componente fisiológico y emocional, lo cual es difícil medir en la clínica, pero es fácilmente reconocido. Puede afectar la calidad del sueño y la salud mental y, en general, tiene un impacto negativo en la motivación del paciente; está estrechamente relacionado con la disnea, así como con la edad, enfermedades crónicas e inactividad. Los programas de ejercicios permiten una mayor actividad física en estos pacientes, logrando un menor agotamiento físico y emocional.

Los cuidados paliativos son un abordaje comprensivo a largo plazo que puede ser paralelo o integrado al tratamiento del cáncer, mientras que la rehabilita- 
ción pulmonar puede ser instaurada para resolver problemas específicos. No todos necesitan rehabilitación pulmonar, pero la mayoría se beneficia de los cuidados paliativos y de soporte. La rehabilitación pulmonar se enfoca en habilidades y técnicas para maximizar la capacidad funcional del paciente en la vida; en circunstancias específicas, es razonable integrar la rehabilitación pulmonar en el manejo multidisciplinario (16).

\section{Barreras de la rehabilitación pulmonar en cáncer pulmonar}

A pesar de la evidencia que apoya el rol de la rehabilitación en el manejo de los síntomas del cáncer pulmonar, las necesidades de esta se reconocen inadecuadamente. Varios factores previenen de ofrecerla a estos pacientes. Se han identificado 3 barreras: la primera es la falta de remisión por parte de los oncólogos, la segunda es los tiempos de espera para ingresar a un programa de rehabilitación pulmonar y el acceso a los centros de rehabilitación pulmonar (limitación por recursos económicos o distancias domicilio-centro de rehabilitación), y la tercera es la carga de síntomas y las comorbilidades, que hacen difícil completar el programa de rehabilitación pulmonar. Se ha encontrado que menos del $50 \%$ de los pacientes es capaz de completar el programa de ejercicios (3).

La rehabilitación pulmonar intrahospitalaria proporciona los mismos beneficios que los programas ambulatorios, pero la rehabilitación pulmonar intrahospitalaria está limitada por el incremento en los costos (2).

\section{Conclusiones}

La rehabilitación pulmonar es una intervención terapéutica costo-efectiva que, adaptada e individualizada en pacientes con cáncer pulmonar, ha demostrado una mejoría en la tolerancia al ejercicio, reducción de los síntomas y mejoría en la calidad de vida. Las modificaciones conductuales que incluyen cesación de tabaquismo, nutrición adecuada y adherencia a la actividad física proporcionan beneficios sostenibles que se extienden más allá de culminar el programa. Sin embargo, la carga de síntomas, las comorbilidades, la falta de conocimiento de los beneficios y el acceso limitado a los centros de rehabilitación pulmonar son las principales barreras de la rehabilitación pulmonar en aquellos con cáncer pulmonar y otras enfermedades respiratorias crónicas.

\section{Referencias}

1. Global Burden of Disease Cancer Collaboration, Fitzmaurice C, Allen C, Barber RM, Barregard L, Bhutta ZA, et al. Global, Regional, and National Cancer Incidence, Mortality, Years of Life Lost, Years Lived With Disability, and Disability-Adjusted Life-years for 32 Cancer Groups, 1990 to 2015: A Systematic Analysis for the Global Burden of Disease Study. JAMA Oncol. 2017;3(4):524-48.

2. Rivas-Perez H, Nana-Sinkam P. Integrating pulmonary rehabilitation into the multidisciplinary management of lung cancer: a review. Respir Med. 2015;109(4):437-42.

3. Wang H, Liu X, Rice SJ, Belani CP. Pulmonary Rehabilitation in Lung Cancer. PM R. 2016;8(10):990-6.

4. Hill K, Bansal V, Brooks D, Goldstein RS. Repeat pulmonary rehabilitation programs confer similar increases in functional exercise capacity to initial programs. J Cardiopulm Rehabil Prev. 2008;28(6):410-4.

5. Güell MR, Cejudo P, Ortega F, Puy MC, Rodríguez-Trigo G, Pijoan JI, et al. Benefits of Long-Term Pulmonary Rehabilitation Maintenance Program in Patients with Severe Chronic Obstructive Pulmonary Disease. Three-Year Follow-up. Am J Respir Crit Care Med. 2017;195(5):622-9.

6. Spencer LM, Alison JA, McKeough ZJ. Maintaining benefits following pulmonary rehabilitation: a randomised controlled trial. Eur Respir J. 2010;35(3):571-7.

7. Cavalheri V, Tahirah F, Nonoyama M, Jenkins S, Hill K. Exercise training for people following lung resection for non-small cell lung cancer - a Cochrane systematic review. Cancer Treat Rev. 2014;40(4):585-94.

8. Janssen SM, Abbink JJ, Lindeboom R, Vliet Vlieland TP. Outcomes of Pulmonary Rehabilitation After Treatment for Non-Small Cell Lung Cancer Stages I to IIIa: AN OBSERVATIONAL STUDY. J Cardiopulm Rehabil Prev. 2017;37(1):65-71.

9. Quist M, Adamsen L, Rørth M, Laursen JH, Christensen KB, Langer SW. The Impact of a Multidimensional Exercise Intervention on Physical and Functional Capacity, Anxiety, and Depression in Patients With Advanced-Stage Lung Cancer Undergoing Chemotherapy. Integr Cancer Ther. 2015;14(4):341-9.

10. Murphy RA, Clandinin MT, Chu QS, Arends J, Mazurak VC. A fishy conclusion regarding n-3 fatty acid supplementation in cancer patients. Clin Nutr. 2013;32(3):466-7.

11. Saito H, Hatakeyama K, Konno H, Matsunaga T, Shimada Y, Minamiya Y. Impact of pulmonary rehabilitation on postoperative complications in patients with lung cancer and chronic obstructive pulmonary disease. Thorac Cancer. 2017;8(5):451-60.

12. Sebio R, Yáñez-Brage MI, Giménez-Moolhuyzen E, Valenza MC, Reychler G, Cahalin LP. Impact of a pre-operative pulmonary rehabilitation program on functional performance in 
patients undergoing video-assisted thoracic surgery for lung cancer. Arch Bronconeumol. 2016;52(5):231-2.

13. Hashmi A, Baciewicz FA Jr, Soubani AO, Gadgeel SM. Preoperative pulmonary rehabilitation for marginal-function lung cancer patients. Asian Cardiovasc Thorac Ann. 2017;25(1):47-51.

14. Kim SK, Ahn YH, Yoon JA, Shin MJ, Chang JH, Cho JS, et al. Efficacy of Systemic Postoperative Pulmonary Rehabilitation After Lung Resection Surgery. Ann Rehabil Med. 2015;39(3):366-73.
15. Maeda K, Higashimoto Y, Honda N, Shiraishi M, Hirohata T, Minami K, et al. Effect of a postoperative outpatient pulmonary rehabilitation program on physical activity in patients who underwent pulmonary resection for lung cancer. Geriatr Gerontol Int. 2016;16(5):550-5.

16. Tiep B, Sun V, Koczywas M, Kim J, Raz D, Hurria A, et al. Pulmonary Rehabilitation and Palliative Care for the Lung Cancer Patient. J Hosp Palliat Nurs. 2015;17(5):462-8. 